\section{PROTECTIVE EFFECTS OF EPIFRIEDELINOL IN A RAT MODEL OF TRAUMATIC BRAIN INJURY ASSESSED WITH HISTOLOGICAL AND HEMATOLOGICAL MARKERS}

Abstract

Background: This study evaluated the protective effects of epifriedelinol (EFD) in a rat model of traumatic brain injury (TBI). Methodology: TBI was induced by dropping a weight from a specific height. The animals were separated into control, TBI, and EFD 100 and $200 \mathrm{mg} / \mathrm{kg}$ groups. The latter received 100 and $200 \mathrm{mg} / \mathrm{kg}$ EFD, respectively, for 2 days beginning $30 \mathrm{~min}$ after inducing TBI. The neurological examination score, permeability of the blood-brain barrier (BBB), water content of the brain, cytokine levels, and oxidative stress parameters were measured in the rats. The effects of EFD on glial fibrillary acidic protein (GFAP)-positive cells were evaluated using immunohistochemistry. Result: The EFD treatment significantly decreased the neurological score, permeability of the BBB, and water content of brain compared with the TBI group. The levels of interleukin-1 (IL-1 1 ), tumor necrosis factor- $\alpha$ (TNF- $\alpha$ ), and oxidative stress were significantly decreased in the EFD-treated groups. The number of GFAP-positive cells was also significantly reduced in the EFD-treated groups. Conclusion: EFD attenuates the secondary injury in TBI rats by reducing the serum cytokine levels and oxidative stress.

Keywords

- Epifriedelinol $\cdot$ Traumatic brain injury $\cdot$ Interleukin-1 $1 \beta \cdot$ Tumor necrosis factor- $\cdot \cdot$ Oxidative stress
Shiping $\mathrm{Li}^{1}$,

Qiaoying Zhang ${ }^{2}$,

Peiwu $\mathrm{Li}^{1 *}$

Urgent Care Centre,

Lanzhou University Second Hospital,

Lanzhou, Gansu province,

730030, P R. China

${ }^{2} S$ chool of Life Sciences,

Lanzhou University, Lanzhou,

Gansu province, 730030, PR. China

Received 17 November 2017

accepted 20 February 2018

\section{Introduction}

In healthy populations, traumatic brain injury (TBI) has the highest mortality and is the costliest health problem worldwide [1]. TBI can be subdivided into primary and secondary injury. Traumatic events cause the primary injury, while delayed processes associated with trauma lead to secondary injuries, such as the efflux of excitatory neurotransmitters, leading to oxidative stress, proteolysis, ATP depletion, ionic imbalance, and excitotoxicity [2,3].

Several factors contribute to the development of secondary injury following TBI. For example, oxidative stress alters the balance between oxidants and antioxidants present in living cells [4]. The presence of large amounts of polyunsaturated fatty acid in brain cells makes them more sensitive to oxidative stressmediated neuronal damage [5], resulting in increased damage to nucleic acids, proteins, and lipids [6,7]. Endogenous antioxidants can protect against the oxidative stressinduced brain damage either by inhibiting the formation of reactive oxygen species (ROS) or by scavenging ROS [8]. The secondary injury in TBI is due to ischemia, which results from the decreased blood flow after trauma. Moreover, ischemia results in $\mathrm{Na} / \mathrm{K} / \mathrm{ATPase}$ pump failure, since it is sensitive to oxidative stress $[9,10]$. The blood-brain barrier (BBB) breaks down when the activity of the $\mathrm{Na} / \mathrm{K} /$ ATPase pump is altered [11]. Factors such as oxidative stress, myeloperoxidase activity, altered BBB permeability, and cerebral edema play roles in the development of secondary injury following TBI.

However, the secondary injury induced by TBI cannot be managed with existing drugs. Epifriedelinol (EFD), isolated from the roots of Aster tataricus [12], has strong antiinflammatory, antibacterial, antioxidant, and anti-cancer effects $[13,14]$. This study evaluated the protective effects of EFD in TBI.

\section{Material and methods}

\section{Animals}

Albino Wistar rats were acclimatized in the animal facilities for 1 week before the experiment. The animals were housed under controlled conditions as specified in the animal care guidelines. All experimental procedures were approved by the ethics committee of Lanzhou University Second Hospital (IAEC/ LUSH/2017/02).

\section{Experiments}

The rats were divided randomly into four groups. The control group was given vehicle only. The TBI and EFD 100 and 200 groups were subjected to TBI. The EFD groups were given 100 and 200 $\mathrm{mg} / \mathrm{kg}$ EFD, i.p. for 2 days beginning 30 min after induction of the brain trauma.

All animals were anesthetized with chlorpromazine (1 $\mathrm{mg} / \mathrm{kg})$ and ketamine (100 $\mathrm{mg} / \mathrm{kg}$ ) and a midline incision was made after shaving the scalp. Bone wax was used to fix a metallic disc in a central position. The rats were placed on a foam rubber platform and mild trauma was induced by dropping a 300-g steel weight from a height of 1 meter. At the end of the experiment, a neurological examination was performed and hematological and pathological changes were assessed in all animals. 


\section{Neurological examination}

Behavioral and motor changes were assessed using the 20-point neuro score. The behavior assessments included the response to and circling of nociceptive stimuli, postural and walking reflexes, extremity tonus, performance in a smooth climbing platform, and consciousness.

\section{Assessing the BBB permeability}

The integrity of the BBB was evaluated using the Evans Blue (EB) assay. At the end of the experiment, rats were anesthetized with chlorpromazine (1 $\mathrm{mg} / \mathrm{kg})$ and ketamine (100 mg/kg) and EB was injected through the jugular vein. The dye was allowed to circulate for $30 \mathrm{~min}$. Then, saline solution was administered at a pressure of $110 \mathrm{~mm}$ $\mathrm{Hg}$ for $10 \mathrm{~min}$ for transcardial perfusion after opening the chest. The animals were sacrificed by decapitation and the cerebral cortex was isolated from the separated brain. EB-albumin extravasation was quantified in each cortex and protein precipitation was assessed after homogenizing the brain samples for $2 \mathrm{~min}$ with $2.5 \mathrm{~mL}$ of phosphate-buffered saline (PBS) and $2.5 \mathrm{~mL}$ of $60 \%$ trichloroacetic acid. The samples were centrifuged at $1000 \mathrm{rpm}$ for $30 \mathrm{~min}$ after cooling. The absorbance of EB was determined at $620 \mathrm{~nm}$ using a spectrophotometer.

\section{Assessing the brain water content}

The brain was weighed, dried at $100^{\circ} \mathrm{C}$ for 2 days, and reweighed. The brain water content was estimated using the following formula: Percentage of water $=\frac{\text { Wet weight of brain }- \text { Dry weight of brain }}{\text { Wet weight of brain }} \times 100$

\section{Measuring cytokine levels}

The plasma interleukin-1 $\beta$ (IL-1 $\beta$ ) and tumor necrosis factor- $\alpha$ (TNF- $\alpha$ ) concentrations were measured using enzyme-linked immunosorbent assays, as per the manufacturer's instructions.

\section{Measuring oxidative stress markers}

The serum malondialdehyde (MDA) level and superoxide dismutase (SOD) activity were determined using reported methods.

\section{Estimating GFAP using} immunohistochemistry

The animals were anesthetized with an intraperitoneal injection of xylazine $(10 \mathrm{mg} /$ $\mathrm{kg}$ ) and ketamine $(75 \mathrm{mg} / \mathrm{kg})$. Then, $200 \mathrm{~mL}$ of saline was injected into the left ventricle, followed by an equal volume of PBS. The brain tissue was fixed in PBS containing 15-30\% sucrose at $4^{\circ} \mathrm{C}$. Tissue sections were incubated for $48 \mathrm{~h}$ at $4^{\circ} \mathrm{C}$ with a polyclonal anti-rabbit glial fibrillary acidic protein (GFAP) antibody (1:3000). The samples were washed with PBS after incubation and incubated again at room temperature for $1 \mathrm{~h}$ with an anti-rabbit Alexa488 secondary antibody. Finally, the samples were washed several times with PBS and observed using an Olympus IX-81 confocal microscope.

\section{Results}

\section{Effect of EFD on the neurological findings}

Figure 1 shows the effects of EFD on the neuro score in the rat TBI model. The neuro score was significantly $(p<0.01)$ higher in the TB group compared with the controls. However, treatment with EFD significantly $(p<0.01)$ decreased the neuro score in the TBI rats in a dose-dependent manner.

\section{Effect of EFD on the permeability of the BBB}

Figure 2 shows the effects of EFD on the BBB permeability and water content of the brain in the rat TBI model. The EB content and percentage water content in the brain were significantly higher in the TBI group than in the controls. There was a significant decrease in the BBB permeability and water content of the brain in the EFD-treated groups.

\section{Effect of EFD on inflammatory cytokines}

Figure 3 shows the effects of EFD on the serum cytokine levels in the rat TBI model. The TNF- $a$ and IL-1 $\beta$ levels were significantly higher ( $p$ $<0.01$ ) in the TBI group compared with the controls, while they were significantly lower in the EFD-treated groups compared with the TBI group in a dose-dependent manner.

\section{Effects of EFD on oxidative stress}

Evaluating parameters of oxidative stress, the MDA level increased significantly, while SOD activity decreased in the serum of the TBI group compared with the control rats. The EFD treatment significantly decreased the MDA level and increased the SOD activity in the serum of the TBI groups, as shown in Figure 4.

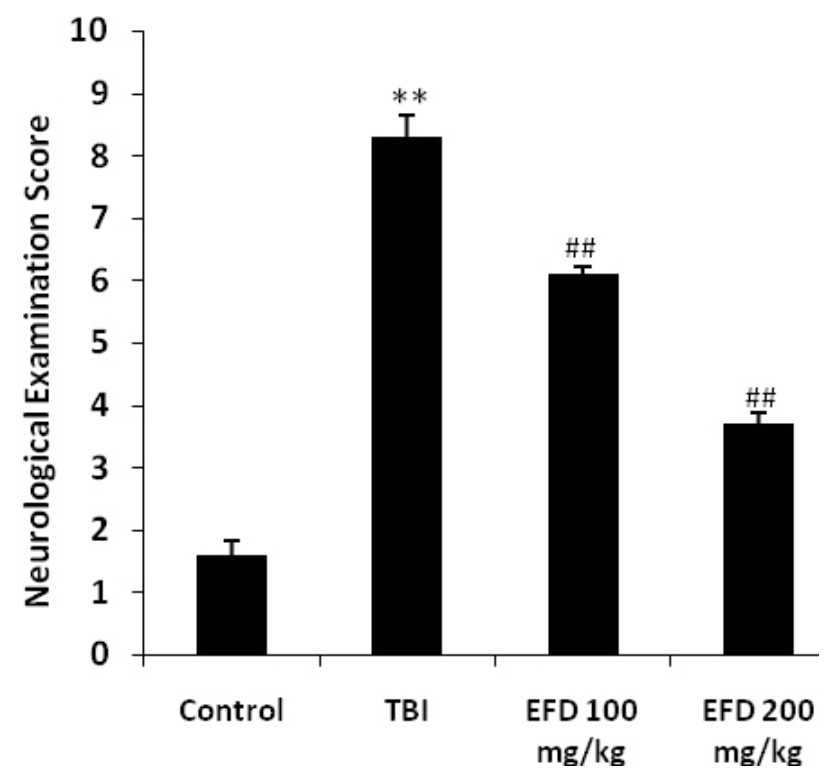

Fig. 1. The effects of epifriedelinol (EFD) on the neuro score in the rat traumatic brain injury (TBI) model. Mean \pm standard deviation (SD) $(\mathrm{n}=10),{ }^{* *} p<0.01$ compared with the controls; ${ }^{* \#} p<0.01$ compared with the TBI group. 

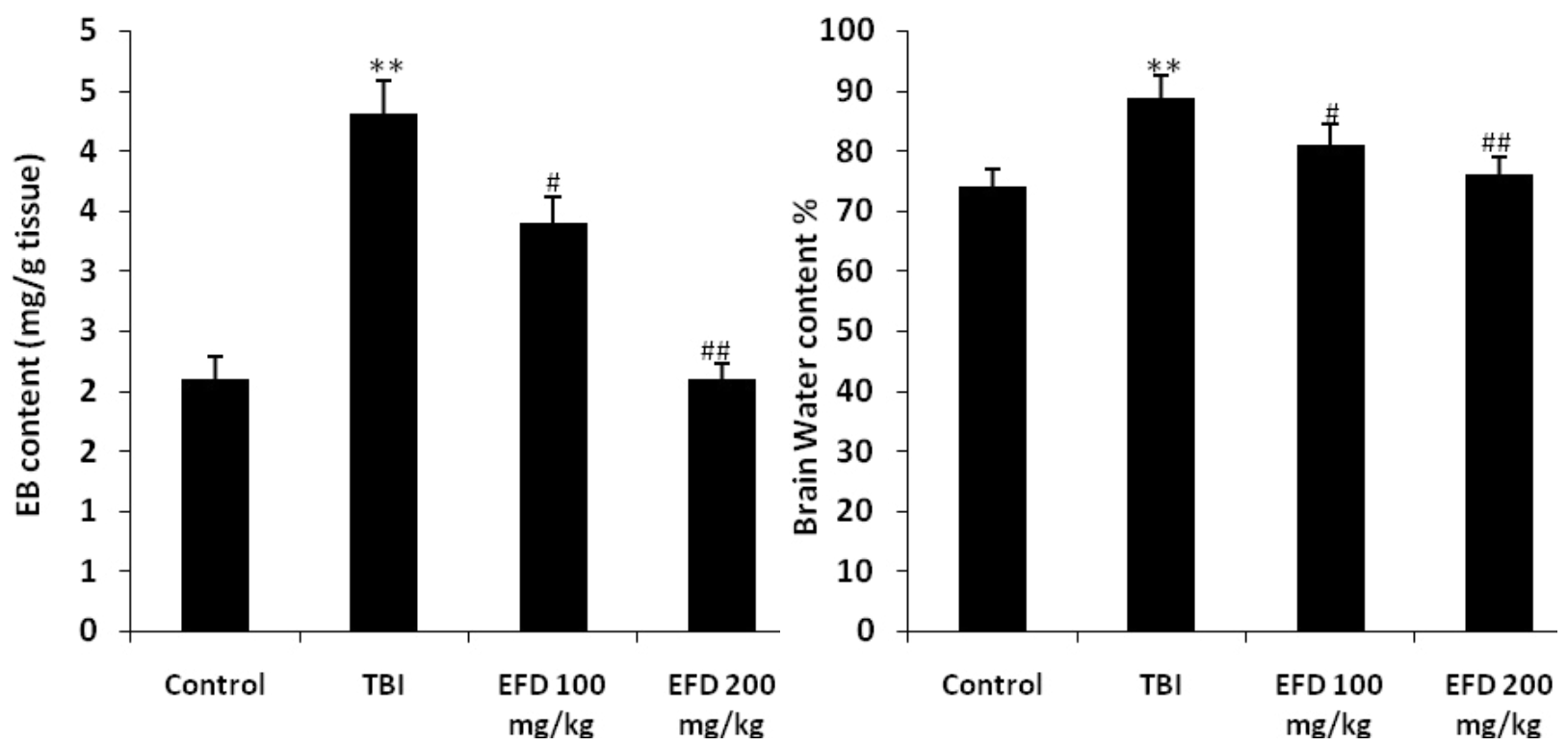

Fig. 2. The effects of EFD on the blood-brain barrier (BBB) permeability and water content of the brain in the rat TBI model. Mean \pm SD $(n=10)$, " $p<0.01$ compared with the controls; $" p<0.05, " \# p<0.01$ compared with the TBI group.
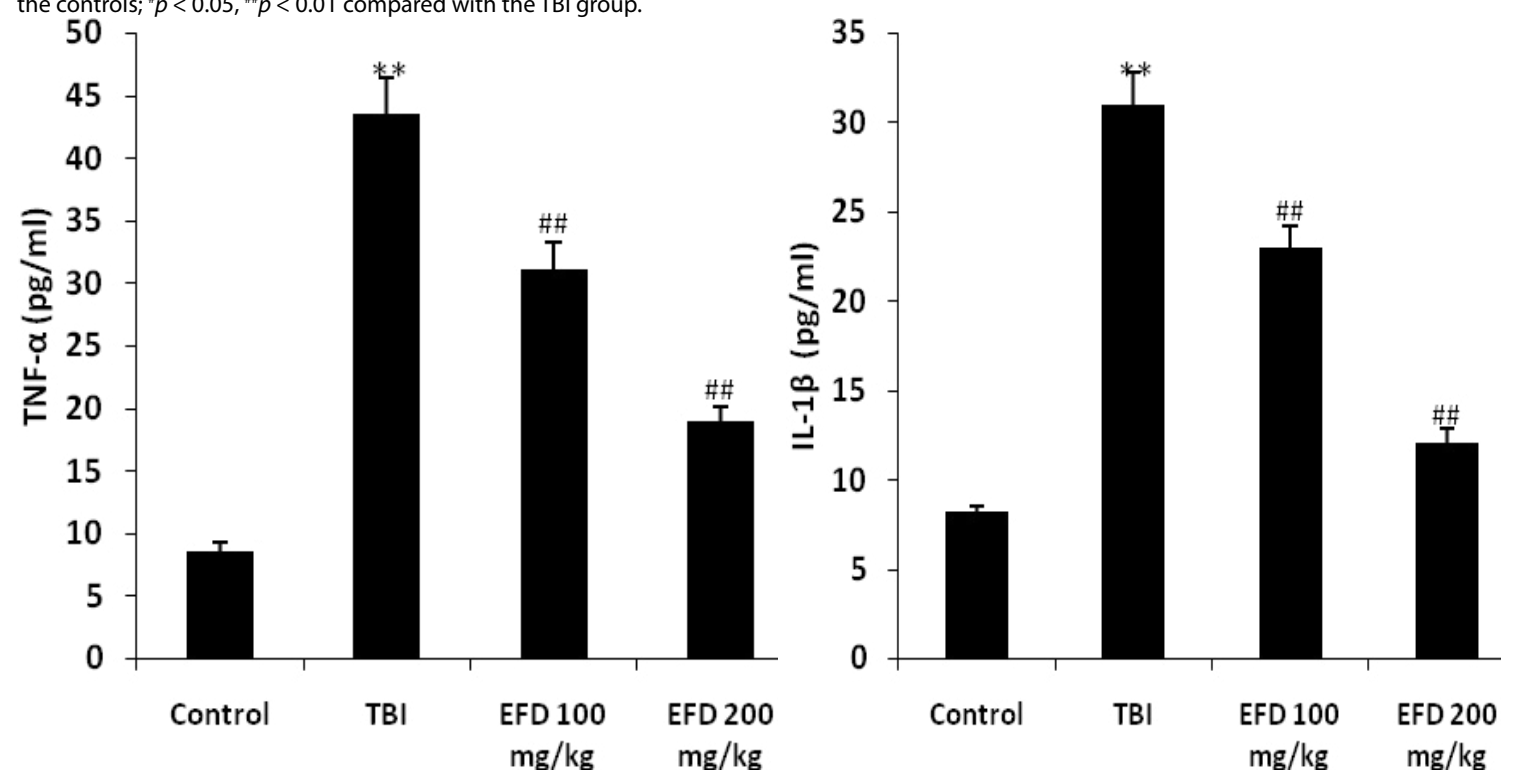

Fig. 3. The effects of EFD on serum tumor necrosis factor- $a$ (TNF- $a)$ and interleukin-1 $\beta$ (IL-1 $\beta)$ levels in the rat TBI model. Mean \pm SD $(n=10)$, " $p<0.01$ compared with the controls; ${ }^{* \#} p<0.01$ compared with the TBI group.

\section{Effect of EFD on GFAP-positive cells}

Figure 5 shows the effects of EFD on GFAPpositive cells in the hippocampus of the TBI model rats. The number of GFAP-positive cells was significantly higher in the hippocampus of the TBI group rats compared with the controls. There was a significant decrease in GFAPpositive cells in the hippocampus of the EFDtreated groups compared with the TBI group.

\section{Discussion}

The mechanism of secondary injury in TBI at the molecular level is not completely understood. Therefore, this study examined the molecular pathology of TBI and evaluated the protective effects of EFD. Following trauma, many pathological changes lead to brain edema, decreased blood flow, and ischemia
[15]. Processes responsible for the increase in secondary brain injury include chemokine generation, cytokines, the production of adhesion molecules, inflammation, and the generation of free radicals [16]. In TBI, the increased cytokine levels cause BBB dysfunction, cell death, and cerebral damage [17]. The neuroinflammation in TBI is promoted by IL-1, which stimulates proinflammatory factors like 


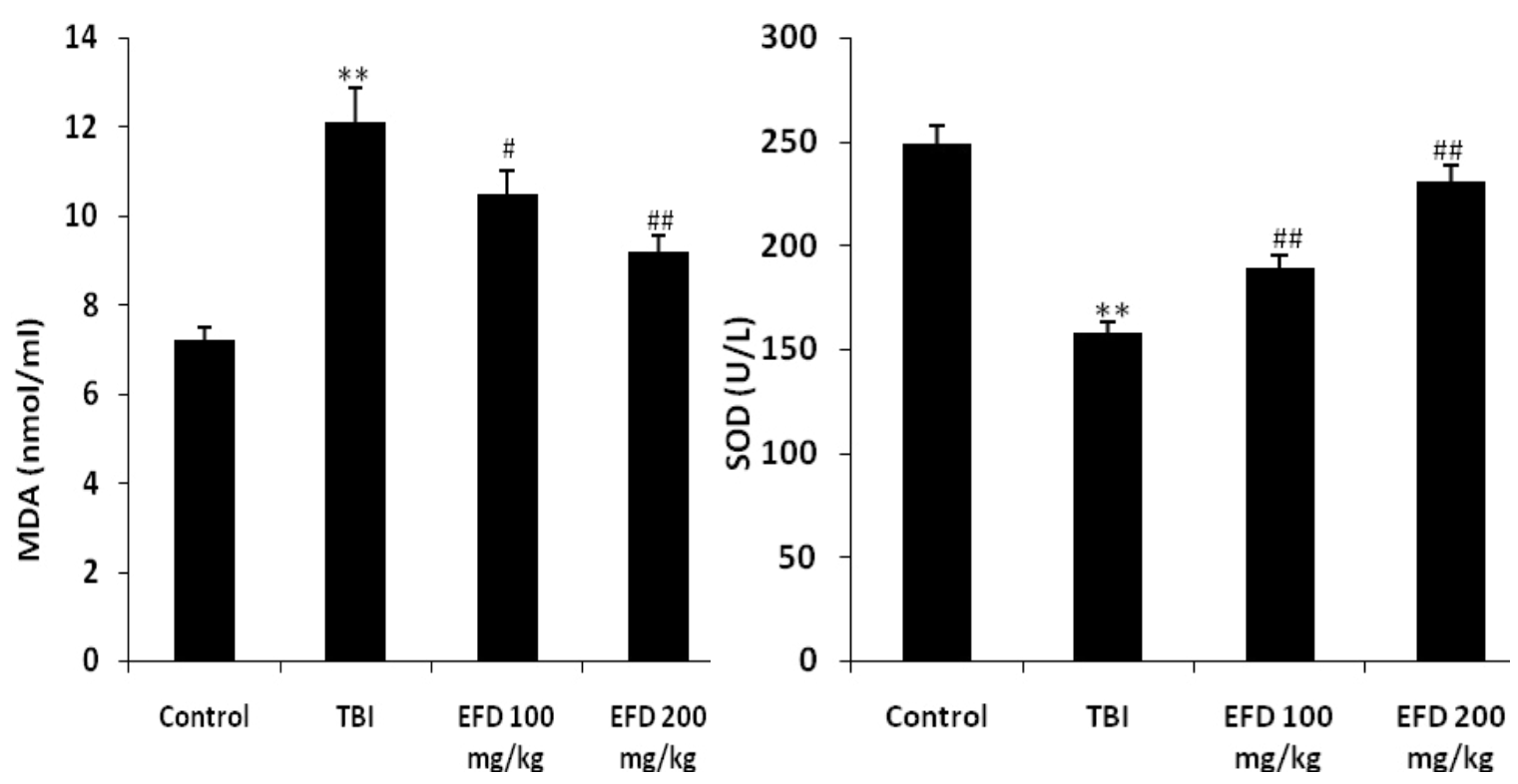

Fig. 4. The effects of EFD on oxidative stress parameters in the serum of TBI model rats. Mean $\pm S D(n=10), " * p<0.01$ compared with Control; $\# p<0.01$ compared with TBI.
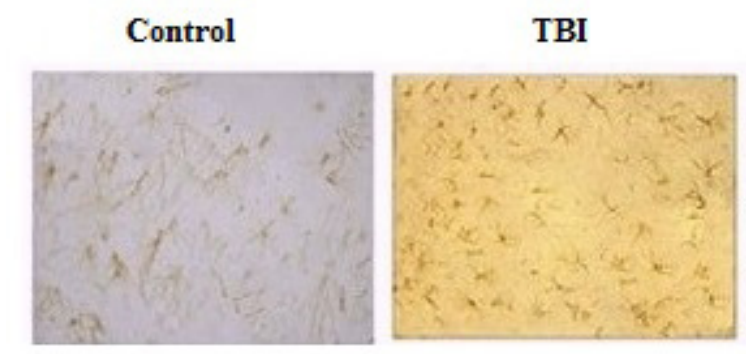

EFD $100 \mathrm{mg} / \mathrm{kg}$

EFD $200 \mathrm{mg} / \mathrm{kg}$

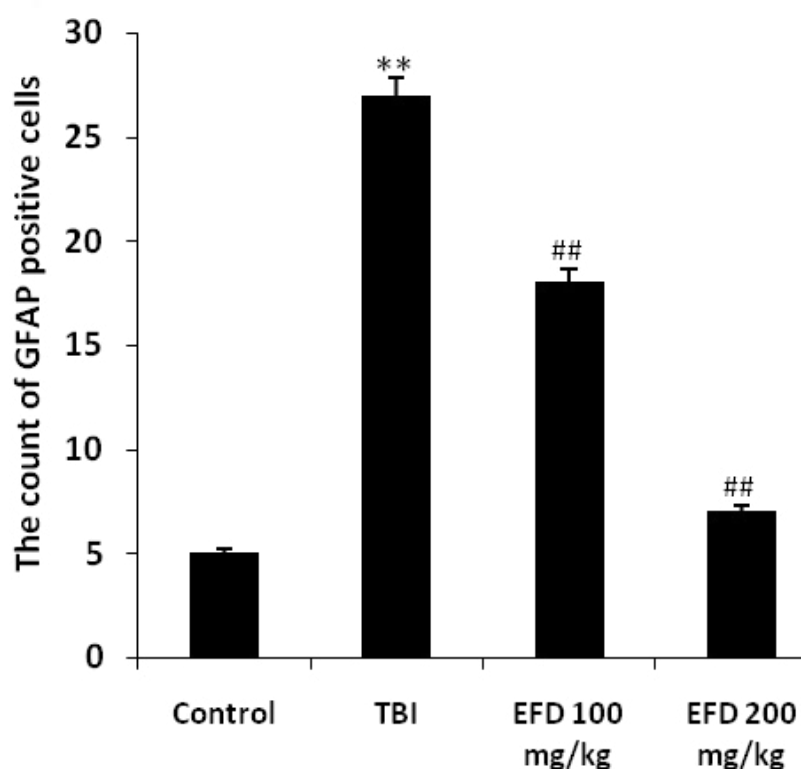

Fig. 5. The effects of EFD on GFAP-positive cells in the hippocampus of the TBI model rats. Mean $\pm S D(n=10), "{ }^{* *} p<0.01$ compared with the controls; ${ }^{* \#} p<0.01$ compared with the TBI group. 
TNF-a $[18,19]$. Hyperinflammation develops because of the presence of proinflammatory mediators in circulation. Our data revealed that treatment with EFD decreased the level of inflammatory mediators in the blood of TBI rats compared with the control group. Moreover, it maintained the integrity of the BBB and water content in the brains of the TBI rats.

Oxidative stress contributes to the development of secondary injury in TBI and antioxidants have a protective effect [20].
Our results suggest that treatment with EFD attenuates oxidative stress in the serum in TBI and decreases the number of GFAP-positive cells in the brain.

\section{Conclusions}

EFD attenuates the secondary injury in TBI in rats by ameliorating the altered serum cytokine levels and oxidative stress.

\section{Acknowledgments}

The authors thank Lanzhou University Second Hospital, China for funding this study.

References

[1] Thurman D, Guerrero J. Trends in hospitalization associated with traumatic brain injury. JAMA 1999; 282: 954-957.

[2] Biegon A, Fry P, Paden C, Alexandrovich A, Tsenter J, et al. Dynamic changes in N-methyl-D-aspartate receptors after closed head injury in mice: implications for treatment of neurological and cognitive deficits. Proc Natl Acad Sci USA 2004; 101: 5117-5122.

[3] Sullivan P, Keller J, Mattson M, Scheff S. Traumatic brain injury alters synaptic homeostasis: implications for impaired mitochondrial and transport function. J Neurotrauma 1998; 15: 789-798.

[4] Shao C, Roberts K, Markesbery W, Scheff S, Lovell M. Oxidative stress in head trauma in aging. Free Radic Biol Med 2006; 41: 77-85.

[5] Ansari M, Ahmad A, Ahmad M, Salim S, Yousuf S, et al. Selenium protects cerebral ischaemia in rat brain mitochondria. Biol Trace Elem Res 2004; 101: 73-86.

[6] Ozdemir D, Uysal N, Gonenc S, Acikgoz O, Sonmez A, et al. Effect of melatonin on brain oxidative damage induced by traumatic brain injury in immature rats. Physiol Res 2005; 54: 631-637.

[7] Reiter R, Tan D, Manchester L, Qi W. Biochemical reactivity of melatonin with reactive oxygen and nitrogen species. A review of the evidence. Cell Biochem Biophys 2001; 34: 237-256.

[8] Biswas K, Bandyopadhyay U, Chattopadhyay I, Varadaraj A, Ali E, et al. A novel antioxidant and antiapoptotic role of omeprazole to block gastric ulcer through scavenging of hydroxyl radical. J Biol Chem. 2003; 278:10993-1001

[9] Kanazawa H, Okada A, Matsushima Y, Yokota H, Okubo S, et al. Determination of omeprazole and its metabolites in human plasma by liquid chromatography-mass spectrometry. J. Chromatogr. A. 2002; 949: 1-9.

[10] Nakagawa S, Arai Y, Kishida T, Hiraoka N, Tsuchida S, et al. Lansoprazole inhibits nitric oxide and prostaglandin $\mathrm{E}(2)$ production in murine macrophage RAW 264.7 cells. Inflammation. 2012; 6: 1062-1068.
[11] Rai K, Matsui H, Kaneko T, Nagano Y, Shimokawa O, et al. Lansoprazole inhibits mitochondrial superoxide production and cellular lipid peroxidation induced by indomethacin in RGM1 cells. J Clin Biochem Nutr. 2011;49:25-30.

[12] Ng TB, Liu F, LuY, Cheng C, Wang Z. Antioxidant activity of compounds from the medicinal herb Aster tataricus. Comp Biochem Physiol: Toxicol Pharmacol 2003; $136: 109-15$.

[13] Kannathasan K, Senthilkumar A, Venkatesalu V. Crystal structure and antibacterial evaluation of epifriedelinol isolated from Vitex peduncularis Wall. ex Schauer. Arab. J. Chem. 2015 doi.org/10.1016/j. arabjc.2015.02.013

[14] Duke J, Ayensu. E. Medicinal Plants of China Reference Publications, Inc. 1985 ISBN 0-917256-20-4

[15] Ansari M, Roberts K, Scheff S. Oxidative stress and modification of synaptic proteins in hippocampus after traumatic brain injury. Free Radic Biol Med 2008; 45: 443-452.

[16] Knoblach S, Fan L, Faden A. Early neuronal expression of tumour necrosis factor-a after experimental brain injury contributes to neurological impairment. J Neuroimmunol 1999; 95: 115-125.

[17] Chen, G, Shi, J, Hu, Z, Hang, C. Inhibitory effect on cerebral inflammatory response following traumatic brain injury in rats: a potential neuroprotective mechanism of $\mathrm{N}$-acetylcysteine. Mediators Inflamm 2008;716458.

[18] Fan L, Young P, Barone F, Feuerstein G, Smith D, et al. Experimental brain injury induces differential expression of tumour necrosis factoralpha mRNA in the CNS. Brain Res Mol Brain Res 1996; 36: 287-291.

[19] Smrcka M, Mrlian A, Karlsson-Valik J, Klabusay M. The effect of head injury upon the immune system. Bratisl Lek Listy 2007; 108: 144-148.

[20] Ozsuer H, Gorgulu A, Kiris T, Cobanoglu S. The effects of memantine on lipid peroxidation following closed-head trauma in rats. Neurosurg Rev 2005; 28:143-147. 